\title{
Existence of Global Solution and Traveling Wave of the Modified Short-Wave Equation
}

\author{
Hyungjin Huh \\ Department of Mathematics, Chung-Ang University, Seoul 06974, Republic of Korea \\ Correspondence should be addressed to Hyungjin Huh; huh@cau.ac.kr
}

Received 9 April 2021; Accepted 26 April 2021; Published 3 May 2021

Academic Editor: Ming Mei

Copyright (C) 2021 Hyungjin Huh. This is an open access article distributed under the Creative Commons Attribution License, which permits unrestricted use, distribution, and reproduction in any medium, provided the original work is properly cited.

The modified short-wave equation is considered under periodic boundary condition. We prove the global existence of solution with finite energy. We also find traveling wave solutions which is the form of elliptic function.

\section{Introduction}

The nonlinear propagation of waves of short wavelength in dispersive systems was discussed in $[1,2]$. They proposed the simple nonlinear short-wave equation that is likely to describe the asymptotic behaviour of the Benjamin-BonaMahony-Peregrine equation [3, 4]. The short-wave equation was studied in [1] numerically for periodic and nonperiodic boundary conditions. The following characteristic initial value problem for the periodic short-wave equation was discussed in [5]:

$$
u_{t x}=u-3 u^{2}
$$

with initial data

$$
u(x, 0)=f(x)
$$

where the real valued function $u$ satisfies the periodic boundary $u(0, t)=u(L, t)$ and describes a small amplitude wave depending on space variable $x$ and time variable $t$.

The solution of the Fourier series was considered in [5]:

$$
u(x, t)=\sum_{n \in Z} u_{n}(t) e^{i 2 \pi n x / L}
$$

where $u_{-n}=\bar{u}_{n}$ for all $n \in Z$. Integrating (1) on $[0, L]$, they obtained

$$
\int_{0}^{L}\left(u-3 u^{2}\right)(x, t) d x=0
$$

which implies, combined with (3),

$$
u_{0}=3 u_{0}^{2}+3 \sum_{n \in Z, n \neq 0}\left|u_{n}\right|^{2}
$$

Then, they obtained

$$
u_{0}=\frac{1}{6}\left(1 \pm \sqrt{1-36 \sum_{n \in Z, n \neq 0} n^{2}\left|u_{n}\right|^{2}}\right)
$$

from which a restriction on the initial data is imposed to guarantee $\sum_{n \in Z, n \neq 0} n^{2}\left|u_{n}\right|^{2}<1 / 36$. Let us consider a homogeneous solution $u(x, t)=u(t)$. To satisfy (4), possible homogeneous solutions are $u(t)=0$ and $u(t)=1 / 3$ which gives a serious constraint on the initial data.

The present work is motivated by the question of whether the restriction of the initial data can be removed. In order for the initial value problem to make sense for a large range of initial data, it seems to be essential to modify the differential equation (1). We consider the following modified short-wave equation:

$$
u_{t x}=u-3 u^{2}-\frac{1}{L} \int_{0}^{L}\left(u-3 u^{2}\right)(y, t) d y
$$


with initial and boundary conditions

$$
\begin{gathered}
u(x, 0)=f(x), \\
u(0, t)=u(L, t)=g(t),
\end{gathered}
$$

where we assume compatibility condition $f(0)=f(L)=g(0)$. We impose the second condition in (8) to guarantee uniqueness. Note that the initial data (2) only is not sufficient for the uniqueness of solution to the initial value problem [6-11]. Even for the linear equation

$$
v_{t x}=0, \quad v(x, 0)=f(x)
$$

we have solutions $v(x, t)=f(x)+h(t)$, where $h$ is any $C^{1}$ function with $h(0)=0$. For the solution to (7) satisfying (8), we have a compatibility condition, considering $u(0, t)=\sum_{n \in Z} u_{n}(t)$,

$$
u_{0}(t)=g(t)-\sum_{n \in Z, n \neq 0} u_{n}(t)
$$

We refer to Section 2 for more information of (7) and (8).

We consider the solution of Fourier series (3). Let us denote by $H$ the space of complex sequences $v=\left\{v_{n}\right\}_{n \in Z}$ :

$$
H=\left\{v=\left\{v_{n}\right\}_{n \in Z} \mid\|v\|^{2}<\infty, v_{-n}=\bar{v}_{n}\right\}
$$

where the norm is defined by

$$
\|v\|^{2}=\left|v_{0}\right|^{2}+\sum_{n \in Z, n \neq 0} n^{2}\left|v_{n}\right|^{2}
$$

The first result is concerned with the global existence of solution.

Theorem 1. For data $g \in C^{1}[0, \infty)$ and $f \in H$ satisfying $f(0)$ $=g(0)$, problems (7) and (8) have a unique solution $u \in C([$ $0, \infty), H)$ of the form (3).

\section{Remark 2.}

(1) Let $u \in C([0, T], H)$ be a solution of equation (7). Then, we can show several regularity properties by applying the same argument as Proposition 2.3 in [5]. In fact, for all $t \geq 0$, Fourier series solution (3) converges uniformly in $x$. Its sum is differentiable in $x$ for almost all $x \in[0, L]$. The derivative satisfies the condition $u_{x}(\cdot, t) \in L^{2}[0, L]$ and $u_{x}(x, \cdot) \in C[0, \infty)$. Moreover, $u_{x}$ is differentiable in $t$, and (7) holds for almost all $x \in[0, L]$

(2) It is an interesting problem to consider an initial value problem of (1) on the whole line $x \in R$. We refer to $[7,10,11]$ for more information

Our next result is concerned with the existence of the traveling wave solutions of the form

$$
u(x, t)=u(x+c t) .
$$

Note that any constant function $u=C$ is a steady solution of (7). We know, for $L$ periodic function $u$,

$$
\int_{0}^{L} u(x+c t)-3 u^{2}(x+c t) d x=\int_{0}^{L} u(x)-3 u^{2}(x) d x=m
$$

where $m$ is a constant. Substituting the ansatz (13) in (7), we obtain

$$
c \frac{d^{2} u}{d \xi^{2}}=u-3 u^{2}-\frac{m}{L}
$$

where $\xi=x+c t$.

Theorem 3. There are nontrivial traveling wave solutions $u($ $x, t)=u(x+c t)$ to (7) for $m<0$ or $0<m / L<1 / 16$. In fact, we have solutions of the elliptic function

$$
u(x+c t)=a-b s n^{2}(\lambda(x+c t), k) .
$$

We refer to Section 3 for precise values of $a, b, c, \lambda$, and $k$.

With the change of variable $t=1 / 2(T+X)$ and $x=1 / 2($ $T-X)$, equation (1) becomes a semilinear wave equation

$$
u_{T T}-u_{X X}=u-3 u^{2}
$$

and initial condition (2) becomes data on characteristic line $T+X=0$. The Cauchy problem on the torus $T^{n}$ for the semilinear wave equation $v_{t t}-\Delta v+f(v)=0$ with initial data $v(x$ $, 0)=v_{0}(x), v_{t}(x, 0)=v_{1}(x)$ was studied in $[12,13]$. Stability of periodic waves of $\mathrm{KdV}$, Schrödinger, Klein-Gordon equations was studied in [14-16]. It is a quite interesting problem to study the stability or instability of the above traveling wave solution to (7).

In Section 2, Theorem 1 is proved. In Section 3, we find traveling wave solutions of (7) to prove Theorem 3 . We will use $A \lesssim B$ to denote an estimate of the form $A \leq C B$, where $C$ is a positive constant.

\section{Proof of Theorem 1}

Let us introduce the following main result of [5].

Theorem 4. If $f \in H$ satisfies

$$
\begin{gathered}
\sum_{n \in Z, n \neq 0} n^{2}\left|f_{n}\right|^{2}<\frac{1}{72}, \\
\int_{0}^{L} f(x)-3 f^{2}(x) d x=0,
\end{gathered}
$$

then problems (1) and (2) have one and only one solution of the form (3). For all $t \geq 0$, Fourier series (3) converges uniformly in $x$. Its sum is differentiable in $x$ for almost all $x \in[0$ $, L]$. The derivative satisfies the condition $u_{x}(\cdot, t) \in L^{2}(0, L]$ and $u_{x}(x, \cdot) \in C[0, \infty)$. Moreover, $u_{x}$ is differentiable in $t$, and (1) holds for almost all $x \in[0, L]$. 
Two restrictions on the initial data are imposed in Theorem 4. Let us review the derivation of equation (1). The Benjamin-Bona-Mahony-Peregrine equation reads as

$$
V_{t}+V_{x}-V_{x x t}=3\left(V^{2}\right)_{x}
$$

Taking change of variables $V(x, t)=U(\xi, \tau)$ with $\xi=x / \varepsilon$ and $\tau=\varepsilon t$, equation (19) becomes

$$
\varepsilon U_{\tau}+\frac{1}{\varepsilon}\left(U_{\xi}-U_{\xi \xi \tau}-3\left(U^{2}\right)_{\xi}\right)=0
$$

Considering small $\varepsilon$, an asymptotic equation $U_{\xi}-U_{\xi \xi \tau}$ $-3\left(U^{2}\right)_{\xi}=0$ is obtained which can be integrated in $\xi$ to give

$$
U_{\xi \tau}=U-3 U^{2}+H(\tau)
$$

where $H(\tau)$ is integration constant with respect to variable $\xi$. The zero condition $H(\tau)=0$ was considered in [1].

To remove restrictions on the initial data in Theorem 4 and allow more solutions like traveling wave solutions, we consider the following modified short-wave equation:

$$
u_{t x}=u-3 u^{2}-\frac{1}{L} \int_{0}^{L} u-3 u^{2} d x
$$

with conditions

$$
\begin{gathered}
u(x, 0)=f(x), \\
u(0, t)=u(L, t)=g(t),
\end{gathered}
$$

where we assume $f \in H, g \in C^{1}[0, \infty)$ and compatibility condition $f(0)=f(L)=g(0)$. We can check that $\int_{0}^{L} u_{t x} d x=0$ in (22). Note that the initial data (23) only is not sufficient for the uniqueness, and additional condition (24) is needed for the characteristic initial value problem $[6,7,17]$.

Substituting (3) into (22), we obtain a system of ordinary differential equations

$$
\frac{d}{d t} u_{n}=-\frac{i L}{2 \pi n}\left(u_{n}-3 \sum_{\alpha+\beta=n} u_{\alpha} u_{\beta}\right) \text { for } n \neq 0 \text {. }
$$

For $n=0$, the left side of (22) is zero. Let us calculate the right-hand side of (22). Considering the solution of Fourier series (3), we have

$$
\frac{1}{L} \int_{0}^{L} u-3 u^{2} d x=u_{0}-3 u_{0}^{2}-3 \sum_{n \in Z, n \neq 0}\left|u_{n}\right|^{2} .
$$

Then, the right-hand side of (22) becomes for $n=0$

$$
\begin{gathered}
u_{0}-3 \sum_{\alpha+\beta=0} u_{\alpha} u_{\beta}-\frac{1}{L} \int_{0}^{L} u-3 u^{2} d x=u_{0}-3 u_{0}^{2}-3 \sum_{\alpha \neq 0} u_{\alpha} u_{-\alpha} \\
-\left(u_{0}-3 u_{0}^{2}-3 \sum_{n \in Z, n \neq 0}\left|u_{n}\right|^{2}\right)=0,
\end{gathered}
$$

where $u_{-\alpha}=\bar{u}_{\alpha}$ is used. Relation (24) implies

$$
u_{0}(t)=g(t)-\sum_{n \in Z, n \neq 0} u_{n}(t)
$$

Therefore, we arrive at the following system of ODEs:

$$
\begin{gathered}
\frac{d}{d t} u_{n}=-\frac{i L}{2 \pi n}\left(u_{n}-3 \sum_{\alpha+\beta=n} u_{\alpha} u_{\beta}\right) \text { for } n \neq 0, \\
u_{0}(t)=g(t)-\sum_{n \in Z, n \neq 0} u_{n}(t) \\
u_{n}(0)=f_{n}
\end{gathered}
$$

where $f(x)=\sum_{n \in Z} f_{n} e^{i 2 \pi n x / L}$.

We say that a function $u \in C([0, T], H)$ is a solution to problem (22)-(24), if the Fourier coefficients $u_{n}$ satisfy (29) for all $n$. For $v \in C([0, T), H)$, we define an operator $\Phi: C([$ $0, T), H) \rightarrow C([0, T), H)$ :

$$
\begin{gathered}
\Phi_{n}(v)(t)=f_{n}-\frac{i L}{2 \pi n} \int_{0}^{t}\left(v_{n}-3 \sum_{\alpha \in Z} v_{\alpha} v_{n-\alpha}\right) \text { for } n \neq 0, \\
\Phi_{0}(v)(t)=g(t)-\sum_{n \in Z, n \neq 0} \Phi_{n}(v)(t) .
\end{gathered}
$$

We will prove a local existence part of Theorem 1 by applying a standard contraction mapping theorem. Denote by $F_{g} \in C([0, T], H)$ the function $F_{g}(x, t)=f(x)+g(t)$ and consider a space

$$
S_{T M}=\left\{v \in C([0, T], H):\left\|v-F_{g}\right\| \leq M\right\},
$$

where $T>0$ and $M>0$.

Proposition 5. Let $f \in H$ and $g \in C^{1}$. Then, the mapping (30) is a contraction mapping from $S_{T M}$ to $S_{T M}$ for a sufficiently small $T$.

Proof. We follow the argument of Proposition 2.2 in [5] with little modifications which come from the different definition 
of $\Phi_{0}(v)$ in (30) from (6). For $v, \omega \in S_{T M}$, we have

$$
\begin{aligned}
\sum_{n \neq 0} & n^{2}\left|\Phi_{n}(v)-\Phi_{n}(\omega)\right|^{2} \\
\leq & \sum_{n \neq 0}\left(\int_{0}^{t}\left|v_{n}-\omega_{n}\right|+\sum_{k=-\infty}^{\infty}\left|v_{k}-\omega_{k}\right|\left|v_{n-k}\right|+\left|\omega_{k}\right|\left|v_{n-k}-\omega_{n-k}\right| d s\right)^{2} \\
\leq & t \sum_{n \neq 0} \int_{0}^{t}\left|v_{n}-\omega_{n}\right|^{2}+\left(\sum_{k=-\infty}^{\infty}\left|v_{k}-\omega_{k}\right|\left(\left|v_{n-k}\right|+\left|\omega_{n-k}\right|\right)\right)^{2} d s \\
\leq & t \sum_{n \neq 0} \int_{0}^{t}\left|v_{n}-\omega_{n}\right|^{2}+\left|v_{0}-\omega_{0}\right|^{2}\left(\left|v_{n}\right|^{2}+\left|\omega_{n}\right|^{2}\right) \\
& +\left(\sum_{k \neq 0} \frac{1}{k^{2}}\right) \sum_{k \neq 0} k^{2}\left|v_{k}-\omega_{k}\right|^{2}\left(\left|v_{n-k}\right|^{2}+\left|\omega_{n-k}\right|^{2}\right) d s \\
\leq & t\left(\int_{0}^{t} 1+\sum_{n \neq 0}\left|v_{n}\right|^{2}+\left|\omega_{n}\right|^{2} d s\right)\|v-\omega\|^{2} \\
\leq & T^{2}\left(1+\|v\|^{2}+\|\omega\|^{2}\right)\|v-\omega\|^{2} .
\end{aligned}
$$

We also have

$$
\begin{aligned}
\left|\Phi_{0}(v)-\Phi_{0}(\omega)\right|^{2} & \leq\left|\sum_{k \neq 0} \Phi_{k}(v)-\Phi_{k}(\omega)\right|^{2} \\
& \leq\left(\sum_{k \neq 0} \frac{1}{k^{2}}\right)\left(\sum_{k \neq 0} k^{2}\left|\Phi_{k}(v)-\Phi_{k}(\omega)\right|^{2}\right) \\
& \leq C T^{2}\left(1+\|v\|^{2}+\|\omega\|^{2}\right)\|v-\omega\|^{2},
\end{aligned}
$$

where (32) is used. Combining (32) and (33) and considering $v, \omega \in S_{T M}$, we have

$$
\|\Phi(v)-\Phi(\omega)\|^{2} \leq C T^{2}\left(1+\left\|F_{g}\right\|^{2}+M^{2}\right)\|v-\omega\|^{2},
$$

which is contraction mapping for sufficiently small $T>0$.

Let $u \in C([0, T], H)$ be a solution of the equation $u=\Phi($ $u)$. Then, we can show several regularity properties in Remark 2 by applying the same argument as Proposition 2.3 in [5]. We skip the proof. We will prove the conservation of $H$ norm.

Proposition 6. Let $u \in C([0, T], H)$ be a solution of (22). Then, we have

$$
\sum_{n \in Z} n^{2}\left|u_{n}(t)\right|^{2}=\sum_{n \in Z} n^{2}\left|f_{n}\right|^{2}
$$

Proof. Multiplying $\partial_{x} u$ on both sides of (22) and integrating on $[0, L]$, we have

$$
\begin{aligned}
\frac{d}{d t} \int_{0}^{L} \frac{1}{2}\left|\partial_{x} u\right|^{2} d x= & \int_{0}^{L} \frac{1}{2} \partial_{x}\left(u^{2}\right)-\partial_{x}\left(u^{3}\right) d x-\frac{1}{L} \int_{0}^{L} u \\
& -3 u^{2} d x \int_{0}^{L} \partial_{x} u d x=0,
\end{aligned}
$$

which implies

$$
\int_{0}^{L}\left|\partial_{x} u(x, t)\right|^{2} d x=\int_{0}^{L}\left|\partial_{x} u(x, 0)\right|^{2} d x
$$

Moreover, a direct calculation implies that

$$
\int_{0}^{L}\left|\partial_{x} u(x, t)\right|^{2} d x=\sum_{n \in Z} \frac{4 \pi^{2}}{L} n^{2}\left|u_{n}(t)\right|^{2}
$$

from which we can derive (35).

From Proposition 5, we have a local solution $u \in C([0, T$ ), $H$ ) of (22)-(24) for a sufficiently small $T>0$. By Proposition 6 , we can extend a local solution to a global one which completes the proof of Theorem 1 .

\section{Traveling Waves}

Here, we consider a traveling wave solution to (7) of the form

$$
u(x, t)=u(x+c t)
$$

where a positive constant $c$ will be determined later. Note that we have, for $L$ periodic function $u$,

$$
\int_{0}^{L} u(x+c t)-3 u^{2}(x+c t) d x=\int_{0}^{L} u(x)-3 u^{2}(x) d x=m
$$

where $m$ is a constant. Substituting the ansatz (39) in (7), we obtain

$$
c \frac{d^{2} u}{d \xi^{2}}=u-3 u^{2}-A,
$$

where $\xi=x+c t$ and $A=m / L$. We integrate (41) to obtain

$$
\frac{c}{2}\left(\frac{d u}{d \xi}\right)^{2}=-u^{3}+\frac{1}{2} u^{2}-A u:=h(u) .
$$

We will consider the cases of $0<A<1 / 16$ or $A<0$.

(1) For $0<A<1 / 16, h$ has three distinct real roots $0<\alpha$ $<\beta$, where

$$
\begin{aligned}
& \alpha=\frac{1-\sqrt{1-16 A}}{4}, \\
& \beta=\frac{1+\sqrt{1-16 A}}{4} .
\end{aligned}
$$

Applying change of variable $u=\beta-(\beta-\alpha) z^{2}$, we derive an equation for $z$

$$
\left(\frac{d z}{d \xi}\right)^{2}=\lambda^{2}\left(1-z^{2}\right)\left(1-k^{2} z^{2}\right)
$$


where $\lambda^{2}=\beta / 2 c$ and $k^{2}=(\beta-\alpha) / \beta$. It is well known in [18] that the solution of (44) is given by the elliptic function $z(\xi$ )$=s n(\lambda \xi, k)$. Therefore, we have

$$
u(\xi)=\beta-(\beta-\alpha) s n^{2}(\lambda \xi, k)
$$

Since the period of $\operatorname{sn}^{2}(x, k)$ is $2 \int_{0}^{\pi / 2} d y / \sqrt{1-k^{2} \sin ^{2} y}$, we impose the following condition from which the period of (45) becomes $L$ :

$$
2 n \int_{0}^{\pi / 2} \frac{d y}{\sqrt{1-k^{2} \sin ^{2} y}}=\lambda L \text { for some } n \in N
$$

which can be rewritten as

$$
\begin{gathered}
2 n \int_{0}^{\pi / 2}\left(1-\frac{2 \sqrt{1-16 A}}{1+\sqrt{1-16 A}} \sin ^{2} y\right)^{-1 / 2} d y \\
\quad=\frac{L}{\sqrt{c}}\left(\frac{1+\sqrt{1-16 A}}{8}\right)^{1 / 2} .
\end{gathered}
$$
(47).

For a given $0<A<1 / 16$, the constant $c$ is determined by

(2) For $A<0, h$ has three distinct real roots $\alpha_{1}<0<\alpha_{2}$, where

$$
\begin{aligned}
& \alpha_{1}=\frac{1-\sqrt{1-16 A}}{4}, \\
& \alpha_{2}=\frac{1+\sqrt{1-16 A}}{4} .
\end{aligned}
$$

Applying change of variable $u=\alpha_{2}-\alpha_{2} z^{2}$, we have an equation for $z$

$$
\left(\frac{d z}{d \xi}\right)^{2}=\lambda^{2}\left(1-z^{2}\right)\left(1-k^{2} z^{2}\right)
$$

where $\lambda^{2}=\left(\alpha_{2}-\alpha_{1}\right) / 2 c$ and $k^{2}=\alpha_{2} /\left(\alpha_{2}-\alpha_{1}\right)$. Then, we have

$$
u(\xi)=\alpha_{2}-\alpha_{2} s n^{2}(\lambda \xi, k)
$$

To make the solution (50) $L$ periodic, we impose

$$
\begin{array}{r}
2 n \int_{0}^{\pi / 2}\left(1-\frac{1+\sqrt{1-16 A}}{2 \sqrt{1-16 A}} \sin ^{2} y\right)^{-1 / 2} d y \\
\quad=\frac{L}{2 \sqrt{c}}(1-16 A)^{1 / 4} \text { for some } n \in N .
\end{array}
$$

For a given $A<0$, the constant $c$ is determined by (51).
Remark 7. For $A=0$, we have $c(d u / d \xi)^{2}=u^{2}(1-2 u)$ which can be integrated as

$$
\left|\frac{\sqrt{1-2 u}-1}{\sqrt{1-2 u}+1}\right|=e^{\xi+a / \sqrt{c}}
$$

where $a$ is an integration constant. We know that $u \leq 1 / 2$. If $u\left(\xi_{0}\right)=0$ for some $\xi_{0}$, we have $0=e^{\left(\xi_{0}+a\right) / \sqrt{c}}$ which is a contradiction. So, we have $0<u \leq 1 / 2$ or $u<0$. For a periodic function $u$, we have $u(0)=u(L)$. Then, we obtain $e^{(L+a) / \sqrt{c}}=e^{a / \sqrt{c}}$ which is a contradiction. The similar argument can be applied for the case of $A=1 / 16$ to show that there is not a nontrivial periodic solution $u$.

\section{Data Availability}

No data were used to support this study.

\section{Conflicts of Interest}

The author declares that there are no conflicts of interest regarding the publication of this paper.

\section{Acknowledgments}

This work was supported by the National Research Foundation of Korea (NRF) grant funded by the Korea government (MSIT) (2020R1F1A1A01072197).

\section{References}

[1] S. M. Gama, R. A. Kraenkel, and M. A. Manna, "Short-wave instabilities in the Benjamin-Bona-Mahoney-Peregrine equation: theory and numerics," Inverse Problems, vol. 17, no. 4, pp. 863-870, 2001.

[2] M. A. Manna and V. Merle, "Asymptotic dynamics of short waves in nonlinear dispersive models," Physical Review E, vol. 57, no. 5, pp. 6206-6209, 1998.

[3] T. B. Benjamin, J. L. Bona, and J. J. Mahony, "Model equations for long waves in nonlinear dispersive systems," Philosophical Transactions of the Royal Society of London, vol. 272, no. 1220, pp. 47-78, 1972.

[4] D. H. Peregrine, "Long waves on a beach," Journal of Fluid Mechanics, vol. 27, no. 4, pp. 815-827, 1967.

[5] S. M. Gama and G. Smirnov, "The Cauchy problem for a short-wave equation," Electronic Journal of Differential Equations, no. 8, p. 9, 2009.

[6] A. Alexiewicz and W. Orlicz, "Some remarks on the existence and uniqueness of solutions of the hyperbolic equation $\partial z / \partial \mathrm{x}$ $\partial \mathrm{y}=f(x, y, x,(\partial z / \partial x),(\partial z / \partial y)),, \quad$ Studia Mathematica, vol. 15, no. 2, pp. 201-215, 1955.

[7] A. Cabet, "Local existence of a solution of a semi-linear wave equation in a neighborhood of initial characteristic hypersurfaces," Annales de la Faculté des Sciences de Toulouse Mathématiques, vol. 12, no. 1, pp. 47-102, 2003.

[8] A. Cabet, "Local existence of a solution of a semilinear wave equation with gradient in a neighborhood of initial characteristic hypersurfaces of a Lorentzian manifold," Communications in Partial Differential Equations, vol. 33, no. 12, pp. 2105-2156, 2008. 
[9] M. Dossa and R. Tagne Wafo, "Solutions with a uniform time of existence of a class of characteristic semi-linear wave equations near $S^{+}$," Communications in Partial Differential Equations, vol. 44, no. 10, pp. 940-989, 2019.

[10] O. Jokhadze, "On existence and nonexistence of global solutions of Cauchy-Goursat problem for nonlinear wave equations," Journal of Mathematical Analysis and Applications, vol. 340, no. 2, pp. 1033-1045, 2008.

[11] O. Jokhadze, "Cauchy-Goursat problem for one-dimensional semilinear wave equations," Communications in Partial Differential Equations, vol. 34, no. 4, pp. 367-382, 2009.

[12] J.-M. Delort, "On long time existence for small solutions of semi-linear Klein-Gordon equations on the torus," Journal d'Analyse Mathématique, vol. 107, no. 1, pp. 161-194, 2009.

[13] L. V. Kapitanskii, "The Cauchy problem for a semilinear wave equation. I," Journal of Soviet Mathematics, vol. 49, no. 5, pp. 1166-1186, 1990.

[14] J. C. Bronski, M. A. Johnson, and T. Kapitula, "An index theorem for the stability of periodic travelling waves of Korteweg-de Vries type," Proceedings of the Royal Society of Edinburgh, vol. 141, no. 6, pp. 1141-1173, 2011.

[15] S. Gustafson, S. le Coz, and T. P. Tsai, "Stability of periodic waves of $1 \mathrm{D}$ cubic nonlinear Schrödinger equations," Applied Mathematics Research eXpress, vol. 2017, no. 2, pp. 431-487, 2017.

[16] F. Natali and E. Cardoso Jr., "Orbital stability of periodic standing waves for the logarithmic Klein-Gordon equation," Journal of Mathematical Analysis and Applications, vol. 484, no. 2, p. 123723, 2020.

[17] P. Hartman and A. Wintner, "On hyperbolic partial differential equations," American Journal of Mathematics, vol. 74, no. 4, pp. 834-864, 1952.

[18] F. Bowman, Introduction to Elliptic Functions, Wiley, New York, 1953. 